Forum

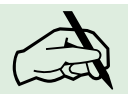

\section{Übersicht über einige Psychotherapien zur Depressionsbehandlung}

Wenn ich die Tabelle 7 der «Übersicht über einige Psychotherapien zur Depressionsbehandlung" [1] richtig interpretiere, dann sind Placebo gleich wirksam wie der «am besten" belegte Kognitive Ansatz und danach sind diese auch der medikamentösen Behandlung «insgesamt überlegen (>2/5)». Da in dieser Tabelle auch der Kognitive Ansatz im Vergleich mit der Kognitiven Therapie ("KT») als "gleich wirksam" hingestellt wird, nehme ich an, dass hier einige Fehler unterlaufen sind.

Ebo Aebischer, Muri

1 Zogg W. Übersicht über einige Psychotherapien zur Depressionsbehandlung. Schweiz Ärztezeitung 2000;81(37):2086-93.

Revue de quelques psychothérapies utilisées dans le traitement des états dépressifs

Si j'interprète correctement le tableau 7 de votre article "Revue de quelques psychothérapies utilisées dans le traitement des états dépressifs" [1], le placebo serait aussi efficace que l'"Approche cognitive», la mieux documentée, et celle-ci serait aussi plus efficace dans l'ensemble $(<2 / 5)$ que le traitement médicamenteux. Si l'approche cognitive est aussi efficace que le placebo et plus efficace que le traitement médicamenteux, il en ressort indirectement que le placebo est plus efficace que le traitement médicamenteux. Cela est-il possible?

Ebo Aebischer, Muri

1 Zogg W. Revue de quelques psychothérapies utilisées dans le traitement des états dépressifs. Bull Med Suisses 2000;81(37):2094-101.

\section{Übersicht über einige Psychotherapien zur Depressionsbehandlung}

Kollege Zogg [1] behauptet u. a. Indikation und Wirksamkeit von vier Psychotherapien und leitet daraus für den orientierungssuchenden (Haus-)Arzt praktische Tips ab. Bei der einzigen erwähnten "psychodynamischen" Therapieform bleibt es bei der Indikations- und Wirksamkeitsbehauptung, bei zwei Therapieformen werden die Behauptungen mit der Studie von K. Grawe (1994) belegt. Die Schlussfolgerungen dieser Studie - von Kollege Zogg beschrieben - wurden von vielen Krankenkassenfunktionären und Politikern dank einer enormen Medienkampagne mit Begeisterung und Genugtuung aufgenommen: Sie schien u.a. den Beweis zu liefern, dass bei psychisch kranken Menschen (und den dahinter stehenden TherapeutInnen) ein grosses, ökonomisches Einsparungspotential liegt wenn die "richtige" Therapie angewandt würde.

Nun ist hinlänglich und ausführlich in Untersuchungen [2] dargelegt worden - allerdings ohne Medienecho -, dass die im (ökonomischen) Zeittrend liegenden, zentralen Behauptungen des versierten Psychotherapieforschers Grawe nicht als faktengeschützt gelten können. Mehr als verunsichernd wirken sich die unhaltbaren Behauptungen auf die Betroffenen (PatientInnen wie TherapeutInnen) aus, die nicht dem (vorgegaukelten) "mainstream" angehören, verheerend aber sind daraus zu ziehende gesundheitspolitische Konsequenzen: Den unterschiedlichen PatientInnen mit ihren vielfältigen Krankheiten entsprechen verschiedene Psychotherapiearten und TherapeutInnen. Einzig aus dem ökonomischen Aspekt und der Übersichtlichkeit heraus eine Uniformität zu verlangen, behindert die kritisch-sachliche Psychotherapieforschung, zerstört aber vor allem eine differenzierte Psychotherapieversorgung der Menschen.

\section{Dr. med. U. Vogel, Oftringen}

1 Zogg W. Übersicht über einige Psychotherapien zur Depressionsbehandlung. Schweiz Ärztezeitung 2000;81(37):2086-93.

2 Fäh M, Fischer G (Hrsg.). Sinn und Unsinn in der Psychotherapieforschung. Giessen: Psychosozial-Verlag; 1998.

\section{Repliken}

Ebo Aebischer, Muri

In der Tabelle 7 hat sich leider effektiv ein Fehler eingeschlichen. Unter "Kognitiver Ansatz», 3. Zeile, wäre "KT» zu streichen. Es hiesse dann: Vgl. VT, IPT, Plazebo: gleich wirksam. Es wäre anzufügen, dass man in der benutzten Studie Plazebo kombiniert mit einer (unspezifischen) Gesprächstherapie verwendete, was ich aus Platzgründen wegliess. Bitte um Entschuldigung.

\section{Dr. med. U. Vogel, Oftringen}

Dass aus der (beanstandeten) Metaanalyse von Grawe Schlussfolgerungen verschiedener Art gezogen wurden, bleibt wohl unumgänglich und liegt in der Natur der Vergleiche. Mein Artikel soll eine Orientierungshilfe sein. Ich bin durchaus der Meinung, dass unterschiedliche (depressive) Patienten und $\mathrm{Pa}$ tientinnen verschiedene Ansprüche, Voraussetzungen und Zielsetzungen haben können und - warum nicht? - verschiedene Therapieformen mit entsprechend ausgebildeten TherapeutInnen in Anspruch nehmen sollen, verlange keine Uniformität, auch nicht ökonomische, lebe gerne mit der Vielseitigkeit in den Psychotherapien.

Dass wir Psychotherapeuten und -therapeutinnen - wie andere Sanitätsberufe - zunehmend unter ökonomischen Druck geraten, liegt wohl nicht an einzelnen Vergleichsstudien verschiedener Therapieformen. Vermehrte Therapieforschung und Qualitätsnachweis werden wohl angesagt sein. Packen wir's!

Dr. med. Walter Zogg, Mendrisio 
Forum

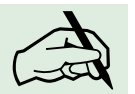

Doppelblindversuche und Evidence-based Medicine - neue Aspekte im Herbst 2000

Samstag, 1. Oktober 2000, morgens etwa um 8 Uhr. Gerade habe ich in unserem Spital zusammen mit dem diensthabenden Assistenzarzt den ersten Morgennotfall versorgt. Beim Frühstück gerät mir neben der Tageszeitung ein Exemplar der Zeitschrift "VISTA" in die Hand. Noch nicht ganz wach blättere ich ein wenig darin herum. Eine Schlagzeile auf Seite 10 springt mir in die Augen: «Doppelblindversuche zur Erprobung neuer Medikamente sind eine flagrante Verletzung des hippokratischen Eides.» [1]

Wir Ärzte sind uns ja in der Zwischenzeit vieles gewohnt: Wir sind überheblich, geldgierig, nur auf unsere Vorteile bedacht, belasten die Krankenkassen mit unserer übertriebenen Diagnostik und Therapie und haben nur eines im Sinn: Mit möglichst wenig Aufwand auf Kosten der kranken Menschheit möglichst rasch möglichst viel Geld $\mathrm{zu}$ verdienen. Meineid - welch ein neuer Aspekt!

Ich lese - jetzt sehr wach - den Artikel des Heinz Joho, der solche starken Ausdrücke zu benutzen weiss. Seine Argumentation kann etwa so zusammengefasst werden: Früher hätte der Doktor etwas verordnet, das vom Apotheker gemixt wurde. Der Patient hätte diese Arznei nach Vorschrift einzunehmen gehabt, obwohl die Mixtur objektiv oft wirkungslos gewesen sei. Die Mischung ArztApotheker-Medikament hätte bereits mindestens die halbe Heilung bedeutet.
Heute hätten wir viel potentere Medikamente, die natürlich auch viel gefährlicher seien. Deshalb müssten unsere neuen Medikamente geprüft werden. Man tue dies mittels randomisierter Doppelblindstudien. Der Patient aber würde über dieses Verfahren meist höchstens unvollständig informiert, wisse also nicht, dass er an einer Studie teilnehme. Er laufe je nach Randomisierung Gefahr, während der Studie völlig ohne Therapie zu bleiben. Trotzdem müsse er respektive die Krankenkasse für die allfällig nicht erhaltene Therapie auch noch bezahlen. Wenn ein Patient in die Plazebogruppe geriete, so erfülle dies den Tatbestand der unterlassenen Hilfeleistung. Dies verletze den hippokratischen Eid ein erstes Mal. Würde ein Patient jedoch in die Verum-Gruppe eingeteilt, wo widerführe im fast noch Schlimmeres, da mit der Erprobung des neuen Medikamentes ja in Kauf genommen werde, dass dem Patienten ein Schaden entstünde. Als Quintessenz formuliert Herr Joho: «Randomisierte Doppelblindstudien zur Erprobung neuer Medikamente sind ethisch fragwürdig."

Ich glaube zuerst an einen Scherz und versuche herauszufinden, worum es sich denn bei "VISTA" wohl handeln könnte. Das Impressum ist wenig ergiebig. Man findet vor allem "Auflage 1,8 Millionen Exemplare" und "wird gratis an alle deutschschweizer Haushaltungen verteilt». Blättert man weiter in "VISTA", so sieht man, dass der redaktionelle Teil etwa 10\%, die Anzeigen etwa 90\% des Heftchens ausmachen. Die Anzeigen preisen praktisch allesamt homöopathische Tropfen und Salben an, die selbstverständlich noch nie in einem Doppelblindversuch beweisen mussten, wie (un?)wirksam sie sind.
Ich gebe die Suche vorläufig auf, um mich dem Wochenenddienst zu widmen. Ich bin trotz der Ermahnungen von Herrn Joho froh, dass ich bei den von mir verordneten Medikamenten weiss, dass sie in sorgfältig geplanten, von ethischen Komitees minutiös überwachten Studien mehrfach auf ihre Wirksamkeit und ihre Nebenwirkungen geprüft wurden.

Wenige Zeit später erscheint in der Tagesschau einer unserer Kollegen, der uns im Nationalrat vertritt. Der Ordinarius einer grossen Universität verkündet uns, dass es nun eine neue Methode gäbe, Medizin fundiert und richtig zu betreiben. Im Ausland sei diese Methode schon lange bekannt - es sei nun an der Zeit, diese in der Schweiz auch einzuführen. Es handle sich um Evidencebased Medicine: «Die Grundlagen für diese neue, richtige Medizin sind Erkenntnisse, die auf randomisierten, kontrollierten Studien und Doppelblindversuchen beruhen."

Wahrlich: Viele neue Aspekte der Evidencebased Medicine innerhalb dieses denkwürdigen Wochenendes: Obwohl einfacher Spitalkardiologe eines Provinzspitals, war ich seit Jahren der Meinung, dass es selbstverständlich sei, seine Medizin auf Evidenz zu basieren. Herr Joho hat mich dezidiert eines besseren belehrt: Wer solches tut, verletzt seinen hippokratischen Eid - und dazu noch flagrant. Von hoher Warte wird mir wenig später bedeutet, dass seit neuem auch in der Schweiz Medizin nur noch evidenzbasiert zu betreiben sei ...

Dr. med. R. Simon, Altdorf

1 Joho H. Doppelblindversuche zur Erprobung neuer Medikamente sind eine flagrante Verletzung des hippokratischen Eides. Vista 2000. S. 10. 